\title{
THE USE OF BOTULINUM TOXIN INJECTION IN THE MANAGEMENT OF MYOFASCIAL PAIN DYSFUNCTION SYNDROME
}

\author{
Nashwa osama*, Sayed Attia** , Mohamed Farmawy ${ }^{* * *}$, Amany Hussien Nemmat" and Mahmoud Shalash*
}

\begin{abstract}
Objectives: To evaluate the short-term effect of the botulinum toxin, type A (BTX-A) injection for the treatment of myofascial pain dysfunction syndrome.

Materials \& Methods: Foueteen female patients suffering from myofascial pain dysfunction syndrome were included in this study. For all patients both the masseter and temporalis muscles were injected once with 20-30 Units of BTX-A respectively and followed up for three months. Follow up included clinical assessment of maximal inter-incisal opening, range of lateral movement and visual analogue scale for pain. Electromyography (EMG) images were ordered pre-operatively and at the end of 3 months' period to quantify the changes in muscle activity.
\end{abstract}

Results: Results of the present study showed resolution of pain in all patients upon completing the follow up period.

Conclusion: Within the limits of this study BTX-A can be used effectively for the treatment of myofacial pain dysfunction syndrome.

\section{INTRODUCTION}

Temporomandibular diseases (TMDs) is a collective term used to describe a number of related disorders affecting the temporomandibular joints, masticatory muscles and associated structures, all of which have common signs and symptoms which includes facial and temporomandibular joint (TMJ) pain, tenderness to palpation on the face and TMJ uncoordinated mandibular movements and the presence of joint sounds. ${ }^{1}$
Myofascial pain syndrome (MPS) is defined as a regional pain disorder of muscular origin characterized by the existence of trigger points within the muscles. The myofascial trigger point (MTrP) is in turn, defined as a palpable and hyperirritable nodule located in a taut band of muscle. Stimulation of these points produces two characteristic phenomena: referred pain and sudden contractions of the taut band, called the local twitch response (LTR). ${ }^{2-3}$

Usual treatment of temporomandibular

\footnotetext{
* Oral Surgery and Medicine Department, National Research Centre, Egypt.

** Professor of Oral \& Maxillofacial Surgery, Cairo University.

*** Lecturer of oral \& Maxillofacial Surgery, Cairo University
} 
myofascial pain includes, pharmacological therapy, splint therapy or both. These two modalities have been shown to produce a temporary relief of painful symptoms. However, long term use of pharmacological treatments can be associated with side effects (gastrointestinal disorders, drug interactions, and adverse reactions) and their use for long periods of time is not recommended. ${ }^{4-6}$

Botulinum toxin (BTX/Botox) is a highly effective bacterial neurotoxin produced by the anaerobic bacterium Clostridium botulinum. Botulinum toxin acts by blocking neuromuscular conduction and produces muscle paralysis, atrophy, and weakness. The toxin has been used extensively for the control of involuntary muscle spasm and for cosmetic treatment. It has also been used for the treatment of hyperfunctional muscles or muscle spasm. ${ }^{5-6}$

This study is aimed to evaluate the effectiveness of intramuscular injections of botulinum toxin for the management of patients who present with myofascial pain dysfunction.

\section{MATERIALS AND METHODS:}

\section{Patients selection:}

A total number of 14 female patients with an age range of 25-55 years were selected from the outpatient clinic of the oral and maxillofacial surgery department, faculty of dentistry, Cairo University. These patients had clinical criteria suggestive of myofascial pain dysfunction syndrome. These criteria were as follows:

- Muscle tenderness in masseter or temporalis muscles or both.

- $\quad$ NO TMJ tenderness.

- Absence of clicking in both temporomandibular joints.

- Absence of any neuromuscular disorders, (E.g: Myasthenia gravis and Lambert-Eaton syndrome).

\section{Pre-Operative Assessment:}

Muscles of mastication (Masseter, Temporalis, Lateral pterygoid, Medial pterygoid), sternocleidomastoid and trapezius were examined for signs of myofascial pain dysfunction syndrome (MPD).

After the initial clinical examination, the following measurements were taken:

- Maximal Inter-incisal Opening. (MIO)

- Range of lateral movements.

- Pain intensity on visual analogue scale. (VAS)

All measurements were recorded in millimeters and tabulated.

\section{Electromyogrphy:}

Selected patients were scheduled for electromyography testing at rest and functional (clenching) position.

Surface electrodes were placed on the masseter and temporalis muscles to gain information about muscle activity during rest and during function. Figure 1.

After palpation of the origin and insertion of the masseter \& temporalis muscles, surface EMG electrodes (Silver/Silverchloride EMG Electrodes, Duo-Trode; Myotronics Inc, Seattle, WA) were placed parallel to the long axis of the muscle, at equal distance from the origin and insertion of the muscle. The anterior border of the anterior temporal muscle was determined by palpation, and surface EMG electrodes were placed behind the frontal process of zygomatic bone, 1.5 to $2 \mathrm{~cm}$ superiorly to the zygomatic arch, perpendicular to the sagittal plane. The patient's skin was cleaned and dried with alcohol before the injections. The patient's head, was positioned according to the Frankfurt horizontal plane parallel to the floor. The EMG records were taken simultaneously from the muscles, Muscle tone was recorded for 5 seconds at rest position, and then for 5 seconds at maximal clenching. The cycle of rest position and maximal clenching was repeated 3 times during each appointment. 


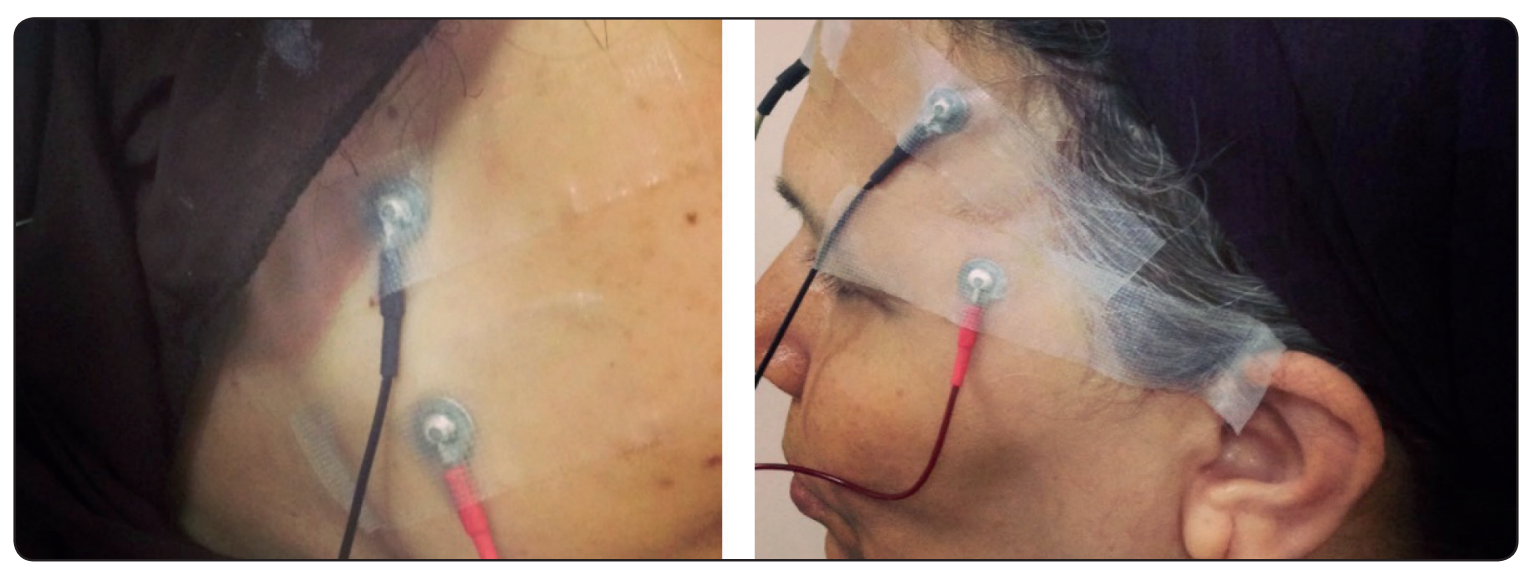

Fig. (1) Showing electrodes of the electromyogram attached to the masseter and temporalis muscles respectively.

\section{Injection Technique:}

Patients were informed and consented on the procedure and on any possible complications that may be encountered such as redness of eyes, pain in site of injection or limited mouth opening.

The BTX vial (Neuronox, Medytox Inc. S. korea) was unpacked and $2 \mathrm{ml}$ saline was added slowly along the walls of the vial to obtain a 5 unit/0.1 ml solution. The solution was carefully swirled to ensure complete mixing. $0.5 \mathrm{ml}$ of solution containing 25 units was drawn into an insulin syringe. 25 Units (U) of BTX-A were injected at the trigger points in masseter and 15 Units (U) of BTX-A were injected in the temporalis muscle.

For each muscle, compression of trigger point technique was performed to confirm the needle position within the trigger point of each target muscle (figure 2). The masseter muscle was approached extraorally with the needle directed towards the trigger points. The temporalis muscle was examined for the trigger points available, once confirmed the needle was inserted through the skin and once touched the trigger point the patients had a painful reflex confirming that the injection was into the trigger point. Aspiration was started to avoid intravascular injection and the solution was injected slowly over period of 5-10 seconds.

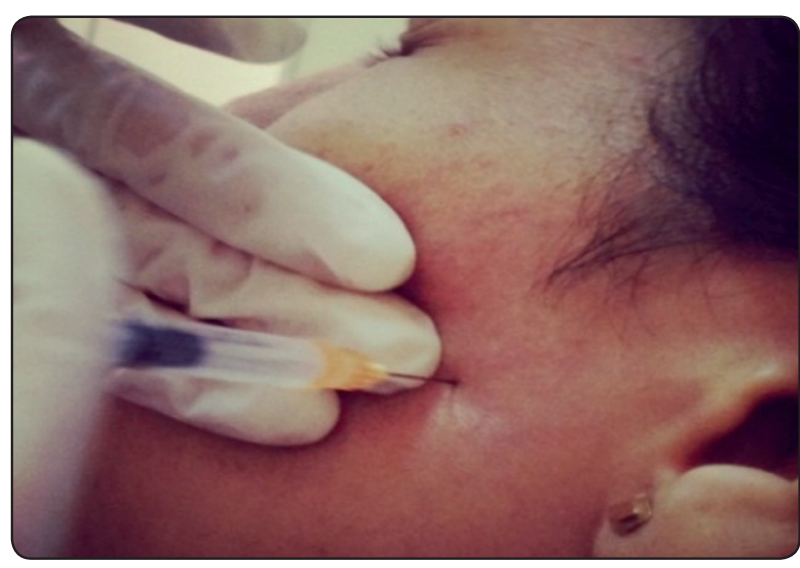

Fig. (2) injection of masseter muscle with BTX.

\section{Post operative instructions:}

Possible side effects were informed to the patient:

- Pain in site of injection which usually subsides within few days.

- Temporary limitation in mouth opening.

- Decreased chewing efficiency.

\section{Follow-up:}

Patients were recalled weekly during the first month then monthly for two months more. The same measurements that were taken pre-operatively were repeated in each of the follow up sessions. EMG readings were ordered at the end of the follow up period. 
The pre and post EMG readings were compared for changes in muscle activity.

\section{Statistical methods:}

The collected data were coded, tabulated, and statistically analyzed using SPSS program (Statistical Package for Social Sciences) software version 18.0.

Descriptive statistics were done for quantitative data as minimum \& maximum of the range as well as mean $\pm \mathrm{SD}$ (standard deviation) for numerical parametric data and median and 1st\& 3rd interquartile range for numerical non-parametric data, while they were done for qualitative data as number and percentage.

Inferential analyses were done for quantitative variables using independent t-test in cases of two independent groups with parametric data and paired t-test in cases of two dependent groups with parametric data. While correlations were done using spearman rho test for numerical non-parametric data.

The level of significance was taken at $\mathrm{P}$ value $<$ 0.050 is significant, otherwise is non-significant. The p-value is a statistical measure for the probability that the results observed in a study could have occurred by chance.

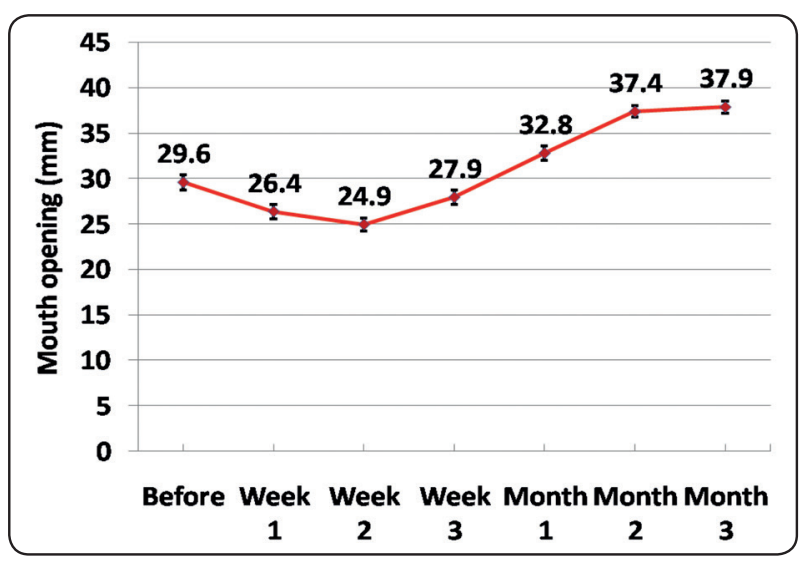

Fig. (3) Mouth opening before and after botulinum toxin injection

\section{RESULTS}

\section{Clinical Findings:}

\section{Range of mouth opening:}

The range of Mouth opening significantly decreased beginning from week 1 till the end of week 2 following Botox injection. This was followed by a significant increase from the beginning of the third week and until the end of the follow up period after 3 months. Figure 3

\section{Range of lateral extrusion:}

The range of Lateral extrusion movements significantly decreased from week 1 till week 2 following Botox injection, then significantly increased at the beginning of week 3 . This continued till the end of the follow up period. Figure 4

\section{Pain Intensity:}

The range of pain intensity on the visual analogue scale (VAS) significantly increased from week 1 following the Botox injection. This was followed by a significant decrease in pain intensity starting from week 2 and continued till the end of the study period. Table 1

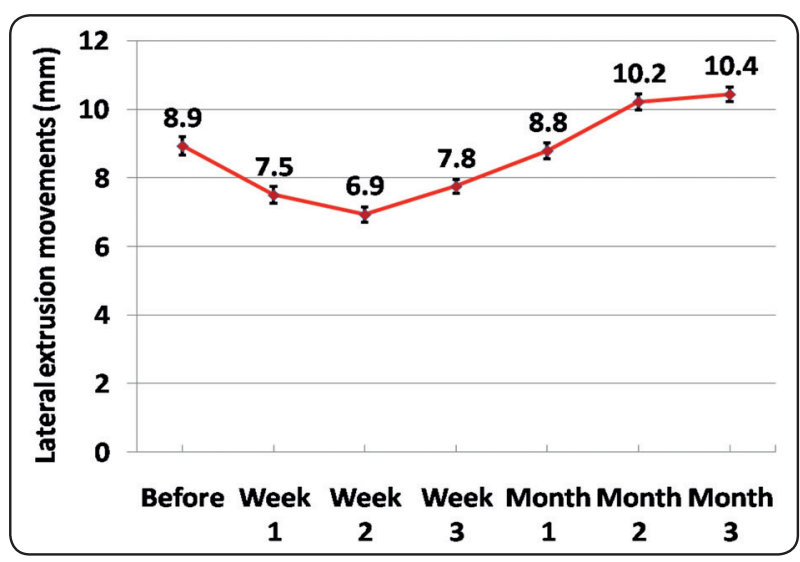

Fig. (4) Lateral extrusion movements before and after botulinum toxin injection 
TABLE (1) Pain intensity changes on the visual analogue scale (VAS) before and after botulinum toxin injection

\begin{tabular}{|l|c|c|}
\hline & Mean \pm SD & Range \\
\hline Before & $8.2 \pm 0.9$ & $7.0-10.0$ \\
\hline Week 1 & $8.9 \pm 0.8$ & $8.0-10.0$ \\
\hline Week 2 & $4.3 \pm 0.7$ & $3.0-5.0$ \\
\hline Month 1 & $3.2 \pm 0.7$ & $2.0-4.0$ \\
\hline Month 2 & $2.1 \pm 0.6$ & $1.0-3.0$ \\
\hline Month 3 & $1.5 \pm 0.7$ & $1.0-3.0$ \\
\hline
\end{tabular}

\section{Electromyogram findings:}

Postoperative analysis of the EMG readings showed significant increase in mouth opening, lateral extrusive movement and significant decrease in pain intensity for different muscle groups treated.

Table 2 \& Figure 5.

Table (2) EMG of resting masseter muscle before and after botulinum toxin injection

\begin{tabular}{|l|c|c|}
\hline & Mean \pm SD & Range \\
\hline Before & $166.5 \pm 45.4$ & $90.8-250.8$ \\
\hline Month 3 & $97.4 \pm 31.3$ & $59.7-162.9$ \\
\hline
\end{tabular}

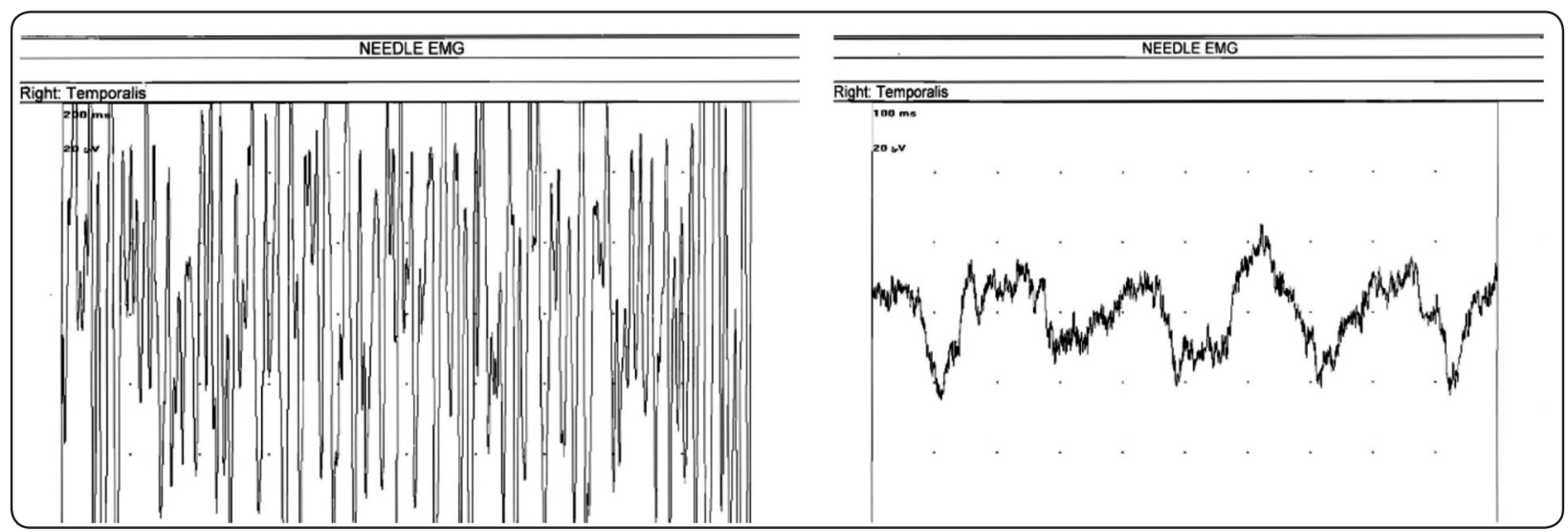

Fig. (5) Showing a decrease in the activity of the right sided temporalis during rest following treatment with botulinum toxin injection at the 3 months follow up.

\section{DISCUSSION}

The Guidelines of the Royal College of Dental Surgeons of Ontario, 2006 clearly stated that conservative treatment should always be the first step in the management of temporo-mandibular disorders and myofascial pain (TMD MP) and that the failure to manage the patient's symptoms with a conservative method does not necessarily imply nor guarantee the success of another more invasive technique. ${ }^{8}$

Several studies have tried to research the effectiveness of different non-invasive techniques. Guided by the promising results of Jose et al ${ }^{7}$, the present study was designed to test the effect of temporalis and masseter muscles injection with BTX on myofascial pain dysfunction syndrome. 
Fourteen female patients were selected for the present study. These patients were complaining from limited mouth opening with pain and tenderness in facial muscles mainly the masseter and temporalis with no tenderness or pain in the TMJ. Patients also showed clinical and EMG imaging evidence of myofascial pain dysfunction syndrome.

In our study a single injection was used. A second injection was to be used only in cases of failure of treatment. The amount of BTX injected into the masseter and temporalis muscles ranged from 20$60 \mathrm{U}$ injected at different points. Final patient recall was done 3 months postoperatively. It is to be noted that the effect of a single injection of BTX declines around the fourth month post injection based on manufacturer recommendations. Normal range of motion (MIO or range of lateral movements) and muscle activity was regained for all patients at the end of the study period. These results are in accordance with the results of Bakke et al ${ }^{10}$

Our results showed that the biggest decline of both mean incisal opening and lateral movements, occurred within the first two weeks following injection and then a gradual regain started on the third week. This complies with the results of a study conducted by Kim et $\mathrm{al}^{11}$.

In our study the VAS was used to assess the effect of BTX-A on the degree of muscle pain. Our results showed improved pain quality score as time progressed, specifically by day 14 . This confirms with the results of previous studies ${ }^{15-16}$ which showed a reduction in TMD pain overtime. This however was inconsistent with the results of another study,${ }^{14}$ which showed that the use of BTX-A was not efficient or cost effective and should not be considered as treatment option for MPDS.

The results of the present study showed that there was a significant improvement in pain for all patients post-operatively. This owes to the paralysis effect of BTX-A through the prevention of acetyl choline release at the motor end neurons. This effect lasts for 2-4 months. In our study the effect lasted
3 months. This is in accordance with the results of the study performed by Kartoglu et al ${ }^{12}$. Absence of pain and the diminished muscle activity within the injected muscles suggests that once the effect of BTX ends, the muscle fibers may stabilize and recover from their hyperactive state.

Generally, the procedure is chair side, well accepted by the patients in absence of local anesthesia. Possible complications may be in the form of a general weakness in the area of the temporomandibular joint which subsides overtime

\section{CONCLUSIONS}

Within the limits of this study, the following may be concluded:

1. BTX-A injection in the temporalis and masseter muscles could be considered a successful treatment option for myofascial pain dysfunction syndrome.

2. Masseter and temporalis muscles appear to be responsible for the myofascial pain dysfunction syndrome.

3. EMG may be used by to assess changes in muscle activity.

4. Further investigation with more patients and extended follow up is advised.

\section{REFERENCES}

1. Conti PCR, Dos santos CN, Koggawa EM, Conti ACF, De Araujo CRP: the treatment of painful temporomandibular joint clicking with oral splints: A randomized clinical trial. J Am Dent Assoc, 137:1108-1114, 2006.

2. Jose M. Climent, Ta-Shen Kuan, Pedro Frenollosa and Fransisco Martin -del- Rosario: Botulinum toxin for the treatment of myofascial pain syndromes involving the nack\&back; A Review from a clinical prespective, 2013.

3. Simons DG: Review of enigmatic MTrPs as a common cause of enigmatic musculoskeletal pain and dysfunction. Journal of Electromyography and Kinesiology. 14(1):95107,2004. 
4. Dolly JO, Aoki KR: The structure and mode of action of different botulinum toxins. European Journal of Neurology.13(supplement 4):1-9,2006

5. Ernberg M, Hedenberg-Magnusson B, List T, Svensson P: Efficacy of botulinum toxin type A for treatment of persistent myofascial TMD pain: a randomized, controlled, double-blind multicenter study. Pain:152(9):19881996.6,2011.

6. Mense S: Neurobiological basis for the use of botulinum toxin in pain therapy. Journal of Neurology, Supplement:251(supplement 1):I1-I7,2004.

7. Jose M. Climent, Ta-Shen Kuan, Pedro Frenollosa and Fransisco Martin -del- Rosario: Botulinum toxin for the treatment of myofascial pain syndromes involving the nack\&back; A Review from a clinical prespective, 2013.

8. Diagnosis \& management of temporomandibular disorders $\&$ related musculoskeletal disorders, Royal college of dental surgeons of Ontario Guidelines of February 2006.

9. Gerwin RD: A review of myofascial pain and fibromyalgia- factors that promote their persistence. Acupunct, 23: 121-134, 2005.

10. Bakke M, Moller E, Werdelin L, Dalager T, Kitai N and kreiborg S. Treatment of severe temporomandibular joint clicking with botulinum toxin in the lateral pterygoid mus- cle in two cases of anterior disc displacement. Oral Surg Oral Med Oral Pathol Oral Radiol Endod, 100:693-700, 2005.

11. Kim K, Byun Y, Kim Y, Kim S. Muscle weakness after repeated injection of Botulinum toxin Type A, evaluated according to bite force measurement of human masseter muscle. Dermatol Surg, 35:1902-7,2009.

12. Kurtoglu C, Gur O, Kurkcu, Sertdemir Y, Guler-Uysal F and Uysal H. Effect of Botulinum Toxin_A in myofscial pain dysfunction patients with or without functional disc displacement. J Oral Maxillofac Surg, 66:1644-51, 2008.

13. Von Linderin JJ, Niederhagen B, Berge S et al: Type A Boulinum Toxin in the treatment of chronic facial pain associated with masticatory hyperactivity. J Oral Maxillofac Surg, 61:774, 2003

14. Nixdorf DR, Heo G, Major PW: Randomized controlled trial of botulinum toxin A for chronic myogenous orofacial pain. Pain 99:465, 2002

15. Freund B, Schwartz M, Symington JM: The use of botulinum toxin for the treatment of temporomandibular disorders: Preliminary findings. J Oral Maxillofac Surg 57:916, 1999

16. Freund B, Schwartz M: Temporal relationship of muscle weakness and pain reduction in subjects treated with botulinum toxin A. J Pain 4:159, 2003 